\title{
Short-Term Propagation Measurements and Modeling for Terrestrial Line-of-Sight Links
}

\author{
Polat Goktas ${ }^{1,2}$, Satilmis Topcu ${ }^{2}$, Ezhan Karasan ${ }^{1}$, and Ayhan Altintas ${ }^{1,2}$ \\ ${ }^{1}$ Department of Electrical and Electronics Engineering, Bilkent University, Ankara, Turkey, \\ e-mail: \{pgoktas, ezhan, altintas\}@ee.bilkent.edu.tr \\ ${ }^{2}$ Bilkent University, Communications and Spectrum Management Research Center (ISYAM), Ankara, Turkey, \\ e-mail: \{pgoktas, topcu, altintas\}@ee.bilkent.edu.tr
}

\begin{abstract}
This paper presents a propagation prediction technique to predict propagation mechanisms for fixed terrestrial line-of-sight (LOS) radio links, especially proposed for rural environments. We report the results of a short-term propagation measurement campaign carried out in the area of Ankara, Turkey. The field measurements were performed at the frequency of $2.536 \mathrm{GHz}$ for a period of three months in summer 2015. It is observed that the difference between measurement data and predicted mean received power is smaller than the standard deviation value provided by Recommendation ITU-R P. 1546.
\end{abstract}

Index Terms - fixed terrestrial line-of-sight (LOS) radio link, field measurement, point-to-point communication, propagation modeling.

\section{INTRODUCTION}

The accurate prediction of propagation mechanisms for terrestrial line-of-sight (LOS) radio links is an essential issue for planning and designing high capacity point-to-point and point-to-multipoint radiocommunication systems. In the planning of terrestrial LOS systems, quantitative data related to the fade margin, mean received power, and worst-month link availability of the channel are required. Usually, link availability of $99.99 \%$ for the worst month is the design target for the fixed terrestrial LOS radio links. In this paper, a method for the prediction of propagation mechanisms for terrestrial LOS links is developed, and it is verified by using short-term propagation measurement data carried out in the area of Ankara, Turkey.

Several empirical propagation prediction models have been proposed over the years for the design of fixed terrestrial LOS radio links, both in clear-air and rainfall conditions. Currently, the Recommendation ITU-R P. 530 [1] is one of the most widely used channel models. This paper provides a modified propagation prediction model for the effects of multipath propagation under the various meteorological conditions. A digital terrain elevation data is used to analyze the path profile between the transmitter (TX) and receiver (RX) sites. The simulation analyses of the modified prediction model are made for fixed terrestrial LOS radio links operating in NATO Band 3+ (1350-2690 MHz) and NATO Band 4 (4400-5000 $\mathrm{MHz}$ ) frequency bands. In addition, modified Bullington model is applied to calculate the diffraction loss for the LOS paths, and a method to calculate reflection points on the terrain profile is developed [2] [3].

The microwave link analyses for several fixed terrestrial microwave LOS/NLOS (line-of-sight/non line-of-sight) radio links are performed using the propagation parameters such as TX and RX station coordinates, path length, frequency, antenna heights above ground level, antenna gains, polarization, radio refractivity gradient, time percentage for attenuation due to rain, rain rate, target SNR (signal-to-noise ratio), bandwidth, digital terrain elevation data, and climate data. A method for the calculation of the received power with the effect of the ground reflection is developed [3] [4]. The implementation of link analysis is verified by using the commercial ATDI ICS telecom software [5] and the short-term field measurement data carried out in the area of Ankara, Turkey.

This paper is organized as follows. In Section II, a brief review of different channel models and the modified propagation prediction model are given. The next section presents information about measurement scenario and comparative study for both measurement data and simulation analyses of the sample LOS radio link. Concluding remarks are given in Section IV, along with the future work.

\section{PROPAGATION PREDICTION MODELING}

In this study, analyses and implementation of propagation prediction models for the design of fixed terrestrial point-topoint systems are provided. Different propagation prediction models in the literature are examined and comparisons are made [3]. Rec. ITU-R P. 530 channel model provided by ITU$\mathrm{R}$ Study Group-3 is analyzed in detail, because it provides the calculation procedure for several propagation mechanisms that must be considered in the design of LOS paths.

The recommendations on the propagation aspects are published in $P$ series of ITU-R. Rec. ITU-R P. 530 "Propagation data and prediction methods required for the design of terrestrial line-of-sight systems" recommendation is one of the most widely used methods providing guidelines for the design of the terrestrial LOS radio link.

The Rec. ITU-R P. 530 channel model is composed of four significant clear-air and rainfall propagation effects relative to free-space for the terrestrial LOS links as follows: 
- Attenuation due to atmospheric gases,

- Attenuation due to rain,

- Fading due to multipath,

- Diffraction loss due to obstruction along the path.

Region-based propagation prediction techniques have been developed for some countries. They include the BarnettVigants model for the United States [6] [7] and the Morita model for Japan [8]. These techniques depend on the frequency, path length and propagation geoclimatic factor predicting the worst-month link availability for LOS paths.

In accordance with the paper by Olsen-Tjelta [9] and Ericssonwide Internal Report [10], the application of three different channel models for many regions around the world clearly shows that the Rec. ITU-R P. 530 channel model gives the best overall performance modelling flat-fading statistics on overland links and also on links in rugged inland regions. The ITU-R propagation database includes 239 links (206 overland and 33 overwater) in 22 countries for frequencies ranging from $450 \mathrm{MHz}$ to $37 \mathrm{GHz}$ located in regions from mountains to overwater. However, this ITU-R propagation database doesn't contain of the propagation data of Turkey region.

A geoclimatic factor is needed to calculate the worst-month link availability for the LOS paths. There are two ways to calculate the geoclimatic factor: a quick calculation $(Q C)$ method, and a detailed link design $(D L D)$ method. The quick calculation method uses only point refractivity gradient in the lowest $65 \mathrm{~m}$ of the atmosphere, $d N_{l}$. However, the detailed link design method uses both $d N_{l}$ and the terrain roughness defined as the standard deviation of terrain heights within a 110 $\mathrm{km} \times 110 \mathrm{~km}$ area, namely $s_{a}$ parameter. The $s_{a}$ data provided by the ITU-R Study Group-3 is too coarse. Therefore, we change the resolution of the terrain roughness data and investigate its effect for the worst-month link availability [11]. The $s_{a}$ data with better resolution improves the accuracy of the analysis. Therefore, the geoclimatic factor of the Rec. ITU-R P. 530 channel model varies based on the refractivity gradient, $d N_{l}$ and terrain roughness, $s_{a}$ parameters in the area of Turkey. However, the other region-based propagation prediction techniques don't depend on these parameters calculating the geoclimatic factor.

Rec. ITU-R P. 530 channel model provides a procedure for estimating the path loss values for clear-air and rainfall conditions. However, the formulation of the received power with the effect of ground reflection along the path is not defined in the Rec. ITU-R P. 530 channel model. In this paper, a method for the calculation of the received power with the effect of the ground reflection is developed [3] [4]. In addition, a modified Bullington model is applied to calculate the diffraction loss for the LOS paths, and a method calculating reflection points on the terrain profile is developed [2] [3].

A free space loss, $L_{F S L}$ in $\mathrm{dB}$, is calculated by using the Friis transmission formula given by [1]:

$$
L_{F S L}=92.45+20 \log (d)+20 \log (f)
$$

where $d$ is the path length in $\mathrm{km}$, and $f$ is the radio frequency in $\mathrm{GHz}$.
The received power formulation related to direct signal (without the effect of the ground reflection) can be calculated as follow [3] [4]:

$$
P_{r}=P_{t x}+G_{t x}+G_{r x}-C L-\left(L_{F S L}+A_{g a s}+A_{\text {rain }}+A_{\text {diff }}\right)
$$

where $P_{t x}$ is the transmitted power in $\mathrm{dBm}, G_{t x}$ and $G_{r x}$ are the gain of TX and RX antennas in $\mathrm{dB}, \mathrm{CL}$ is the cable losses in $\mathrm{dB}, A_{\text {gas }}$ and $A_{\text {rain }}$ are attenuation due to atmospheric gases and rain in $\mathrm{dB}$, and $A_{\text {diff }}$ is the diffraction loss for the terrain profile in $\mathrm{dB}$.

The received power formulation with the effect of the ground reflection in $\mathrm{dB}$ can be calculated as follow [3] [4]:

$$
P_{\text {total }}=10 \log \left(10^{\frac{P_{r}}{10}} \times\left(1-10^{-\frac{\min \left\{L_{s}\right\}}{20}}\right)\right)
$$

where $L_{s}$ are all possible reflection losses along the path in $\mathrm{dB}$.

The fade margin is computed using the following expression [3] [4]:

$$
A=P_{\text {total }}-P_{N}-S N R
$$

where $P_{N}$ is the noise level in $\mathrm{dBm}$, and $S N R$ is the target signal-to-noise ratio in $\mathrm{dB}$.

The calculation procedure of the worst-month link unavailability for detailed link design method is stated as follows [1]:

As a first step, the propagation geoclimatic factor and path inclination are calculated using the following relations:

$$
\begin{gathered}
K=10^{-4.4-0.0027 d N_{1}}\left(10+s_{a}\right)^{-0.46} \\
\left|\varepsilon_{p}\right|=\frac{\left|h_{t x}-h_{r x}\right|}{d}
\end{gathered}
$$

where $h_{t x}$ is the altitude of the transmitting antenna in meters, $h_{r x}$ is the altitude of the receiving antenna in meters.

Finally, the average worst-month link unavailability, $p_{w}$, is calculated as a function of the calculated fade margin, terrain roughness, propagation geoclimatic factor, path length, and frequency parameters given by:

$$
\begin{gathered}
p_{o}=K d^{3.4}\left(1+\left|\varepsilon_{p}\right|\right)^{-1.03} f^{0.8} \times 10^{-0.00076 h_{L}} \\
p_{w}=p_{o} \times 10^{-\frac{A}{10}}
\end{gathered}
$$

\section{MEASUREMENT SET-UP AND RESULTS}

Short-term propagation measurements were carried out for a period of three months in 2015 (June-August, 2015) in the area of Ankara, Turkey. The measurement scenario was performed at the frequency of $2.536 \mathrm{GHz}$ for the case of vertical polarization. As a result of the high directive gain of the antennas used at each of LOS stations, accurate pointing is required. The distance between the two stations is $10.652 \mathrm{~km}$. An aerial photograph of the $10.652 \mathrm{~km}$ terrestrial LOS radio link is given in Fig. 1. A path profile between TX and RX stations is generated using Digital Terrain Elevation Data (DTED-1) with a spatial resolution of $3 \times 3$ seconds. Detailed information regarding the terrestrial LOS path is provided in Table I. The terrain profile of the LOS radio link is presented in Fig. 2. The blue and red curves indicate the first Fresnel zone and 0.6 first Fresnel zone, respectively. 
Received signal levels obtained during the measurements were recorded periodically using N9912A Fieldfox Handheld RF Combination Analyzer onto a computer. The received signal level measurement data for $10.652 \mathrm{~km}$ terrestrial LOS radio link is given in Fig. 3. Based on the data presented in Fig. 3, received power values have a periodic cumulative distribution for the $10.652 \mathrm{~km}$ terrestrial LOS radio link. The comparative study for both measurement data and simulation analyses of the sample LOS radio link as a function of fade depth is given in Fig. 4. In the case of small fade depth, the mean and standard deviation of discrepancies between measured and predicted values for the LOS path is small. In Rec. ITU-R P. 1546 model [12], the standard deviation of a digital signal at $2 \mathrm{GHz}$ frequency is given as $5.5 \mathrm{~dB}$ for certain planning situations. Therefore, the difference between measurement data and predicted received power values are smaller than the standard deviation provided by Rec. ITU-R P. 1546, see Fig. 4.

TABLE I

Terrestrial link and station parameters for the sample LOS radio link.

\begin{tabular}{|l|c|}
\hline Parameters & Description \\
\hline Path length & $10.652 \mathrm{~km}$ \\
\hline Altitude of transmitter station (a.s.l) & $891 \mathrm{~m}$ \\
\hline TX antenna height (a.g.l) & $25 \mathrm{~m}$ \\
\hline Altitude of receiver station (a.s.l) & $1015 \mathrm{~m}$ \\
\hline RX antenna height (a.g.l) & $15 \mathrm{~m}$ \\
\hline Radio frequency & $2.536 \mathrm{GHz}$ \\
\hline Transmitted power & $30 \mathrm{dBm}$ \\
\hline TX/RX antenna gain & $25 \mathrm{dBi}$ \\
\hline TX/RX cable loss & $3.625 \mathrm{~dB}$ \\
\hline Diffraction method & Delta-Bullington \\
\hline Time percentage for rain attenuation & $0.01 \%$ \\
\hline Polarization type & Vertical \\
\hline Ground type & Summer \\
\hline Season type & \\
\hline
\end{tabular}

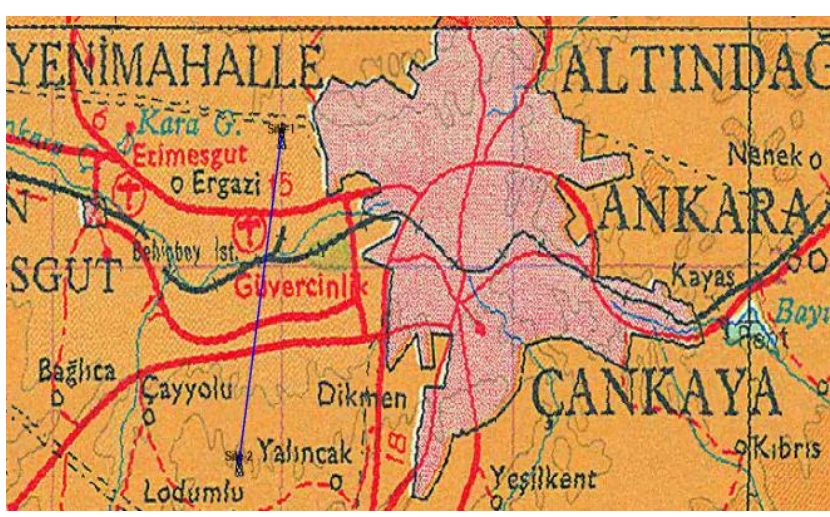

Fig. 1. An aerial photograph of the $10.652 \mathrm{~km}$ terrestrial LOS radio link.

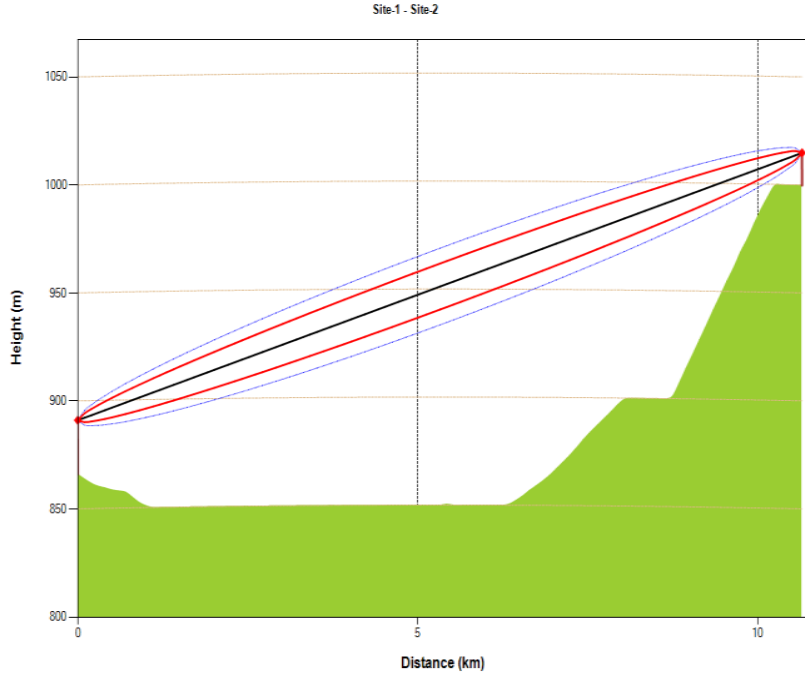

Fig. 2. The terrain profile of the $10.652 \mathrm{~km}$ terrestrial LOS radio link.

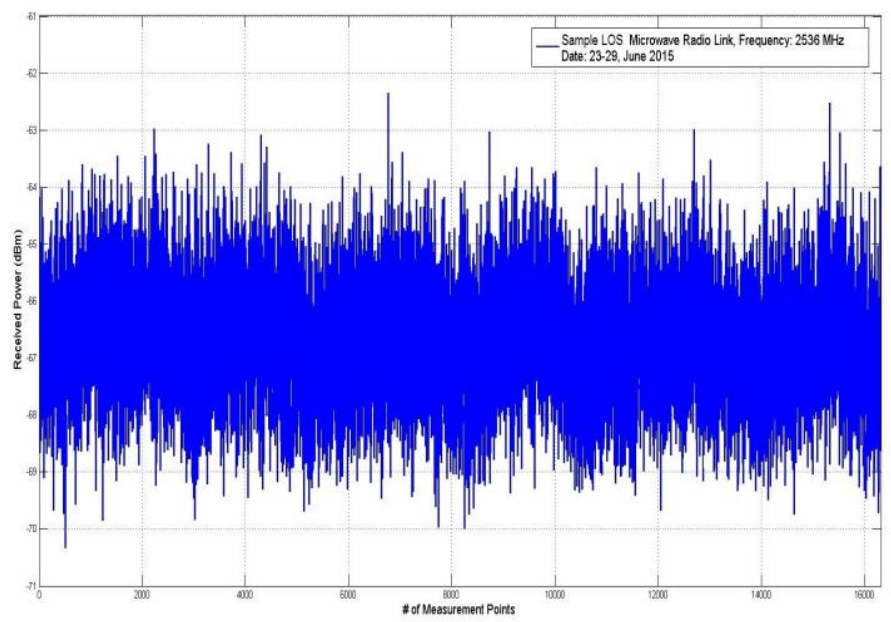

Fig. 3. Received signal level measurement data for the $10.652 \mathrm{~km}$ LOS radio link during June 2015.

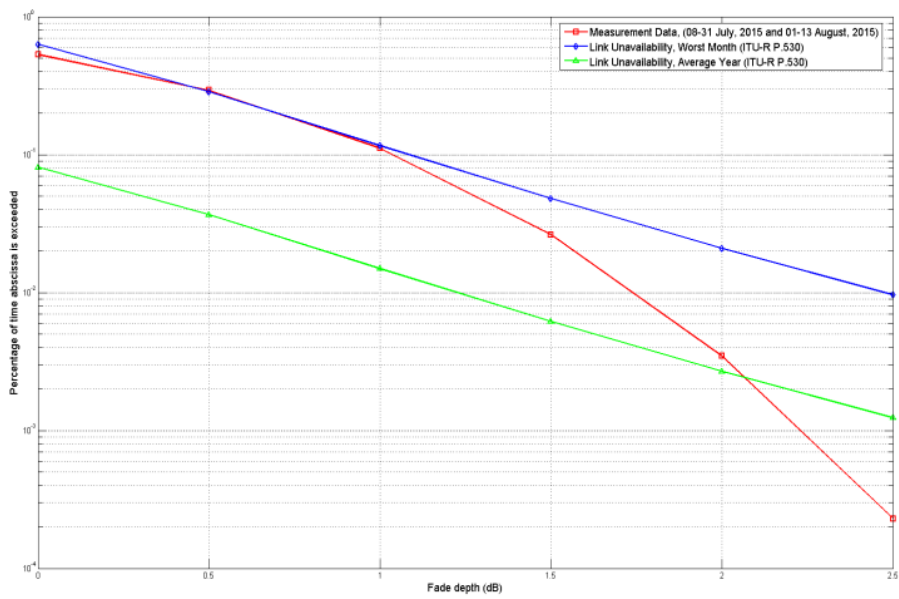

Fig. 4. Comparative study for both measurement data and predicted received power values as a function of fade depth. 


\section{CONCLUSIONS}

This paper presents the results of a short-term propagation measurement campaign carried out in the area of Ankara, Turkey. A modified propagation prediction model for the effects of multipath propagation under the various meteorological conditions has been developed using Digital Terrain Elevation Data. The performance analysis in terms of the received power have been done for sample terrestrial microwave LOS radio links. Furthermore, we have introduced the measurement set-up and the corresponding measurement scenario carried out in the area of Ankara, Turkey. Based on the measurement results, we have provided a comparison between the measurement data and the predicted received power values. It can be shown that the difference between measurement data and predicted received power values are smaller than the standard deviation provided by Rec. ITU-R P. 1546.

\section{ACKNOWLEDGMENT}

This work is supported by ASELSAN Electronic Industries Inc., and the Undersecretariat for Defense Industries (SSM). We wish to acknowledge Mr. Gokhan Moral for his valuable contribution.

\section{REFERENCES}

[1] International Telecommunication Union, "Propagation data and prediction methods required for the design of terrestrial line-of-sight systems", Recommendation ITU-R P. 530-15, 2013.

[2] S. Topcu, P. Goktas, E. Karasan, and A. Altintas, "A new approach to diffraction line-of-sight (LOS) paths", IEEE-APS Topical Conference on Antennas and Propagation in Wireless Communications, Sept. 2015.

[3] P. Goktas, Analysis and implementation of prediction models for the design of fixed terrestrial point-to-point systems. M.S. thesis, Bilkent University, Jan. 2015.

[4] P. Goktas, S. Topcu, E. Karasan, and A. Altintas, "Multipath fading efffect on terrestrial microwave line-of-sight (LOS) radio links", IEEE International Symposium on Antennas and Propoagation and North American Radio Science Meeting, July 2015.

[5] http://www.atdi.com/ics-telecom/.

[6] W. T. Barnett, "Multipath propagation at 4, 6, and $11 \mathrm{GHz}$, Bell Syst. Tech. Journal, vol. 51, pp. 311-361, Feb. 1972.

[7] A. Vigants, "Space diversity engineering", Bell Syst. Tech. Journal, vol. 54, pp. 103-142, Jan. 1975.

[8] K. Morita, "Prediction of Rayleigh fading occurrence probability of line-of-sight microwave links", Rev. Elec. Commun. Lab., vol. 18, pp. 810-821, Dec. 1970.

[9] R. L. Olsen and T. Tjelta, "Worldwide techniques for predicting the multipath fading distribution on terrestrial LOS links: Comparison with regional techniques", IEEE Trans. Antennas Propag., vol. 51, pp. 23-30, Jan. 2003.

[10] K. T. Vieira, "Comparisons of Barnett-Vigants method with ITU-R link data base", tech. rep., EAB/G-03:002609 Uae Ericssonwide Internal Report, August 2003.

[11] P. Goktas, A. Altintas, S. Topcu, and E. Karasan, "The effect of terrain roughness in the microwave line-of-sight (LOS) multipath fading estimation based on Rec. ITU-R P. 530-15", General Assembly and Scientific Symposium (URSI-GASS), August 2014.

[12] International Telecommunication Union, "Method for point-to-area predictions for terrestrial services in the frequency range $30 \mathrm{MHz}$ to 3000 MHz,” Recommendation ITU-R P. 1546-5, Sept. 2013. 